J. DIFFERENTIAL GEOMETRY

52 (1999) $117-144$

\title{
CONVEX BODIES OF CONSTANT BRIGHTNESS AND A NEW CHARACTERISATION OF SPHERES
}

\author{
FRANÇOIS HAAB
}

\begin{abstract}
Nakajima showed that if a convex body in $\mathbb{R}^{3}$ satisfying certain smoothness conditions has constant width and constant brightness, then it is a ball. This work extends Nakajima's result to higher dimensions. We prove that if $K$ is a convex body in $\mathbb{R}^{d}$ of class $C_{+}^{2}$ with constant $i$-brightness and constant $j$-brightness, then $K$ is a ball. We also generalize this result to relative differential geometry.
\end{abstract}

\section{Introduction}

In this article we obtain a new characterisation of balls among convex bodies in $\mathbb{R}^{n}$ of class $C_{+}^{2}$. A convex body $K$ in $\mathbb{R}^{n}$ is of class $C_{+}^{k}, k \geq 2$, if its boundary, denoted by bd $K$, is a hypersurface of class $C^{k}$ and if the Gauss-Kronecker curvature of bd $K$ is positive at any point $x \in \operatorname{bd} K$.

A convex body $K$ in the Euclidean space $\mathbb{R}^{n}$ is of constant $k$-brightness $([10,3.3 .10])$ or of constant outer $k$-measure $([6, p .81])$ if all its orthogonal projections on $k$-dimensional linear subspaces of $\mathbb{R}^{n}$ have the same $k$-volume. When $k=1, K$ is of constant width (Similary when $k=n-1, K$ is of constant brightness).

If $K$ is a convex body in $\mathbb{R}^{n}$ with constant width and constant $k$ brightness for a given $k>1$, is $K$ a ball?

This classical question ([14, p.368]; $[6$, p.82]; [7, problem A10]) is called the Nakajima problem by Goodey, Schneider and Weil in [13]. In 1926, Nakajima [18] answered positively if $n=3$ and $k=2$ and $K$ is of class $C_{+}^{2}$ (see also Bonnesen and Fenchel's book of 1934 ([3, p.140]) or $([10,3.3 .20])$ for a more recent viewpoint $)$.

Received May 15, 1998, and, in revised form, February 23, 1999. 
We will answer this question in the affirmative for convex bodies in $\mathbb{R}^{n}, n \geq 3$, of class $C_{+}^{2}$ where $1<k<n$.

Recently Goodey, Schneider and Weil [12] asked a more general question. It is justified by the fact that every centrally symmetric convex body in $\mathbb{R}^{n}$ is determined by two of its projection functions. Recall that the ith projection function $(1 \leq i<n)$ of a convex body $K$ in $\mathbb{R}^{n}$ is defined on the Grassmann manifold $G(n, i)$ of $i$-dimensional subspaces of $\mathbb{R}^{n}$. Its value at $E \in G(n, i)$ is equal to the $i$-dimensional volume of the orthogonal projection of $K$ on $E$.

If $K$ is a convex body in $\mathbb{R}^{n}$ with constant $i$-brightness and $j$-brightness $(1 \leq i<j<n)$, is $K$ a ball?

Our main theorem gives a positive answer to this second question (and therefore to the first one) with some differentiability conditions:

Theorem A. Let $0<i<j<n$ be integers and $K$ a convex body in $\mathbb{R}^{n}$ of class $C_{+}^{2}$. If $K$ has constant $i$-brightness and $j$-brightness, then $K$ is a ball.

It is not possible to weaken the hypothesis by only considering one type of brightness. In fact, one must remember that for all $n \geq 2$ and $1 \leq k \leq n-1$, there are non-spherical convex bodies in $\mathbb{R}^{n}$ with constant $k$-brightness $([10,3.3 .15-16])$. However, every convex body with constant $k$-brightness which is centrally symmetric is necessarily $([10,3.3 .11])$ a ball, by Aleksandrov's projection theorem $([10,3.3 .6])$.

Note that the first question is open, even in $\mathbb{R}^{3}$, without differentiability assumptions on the boundary ([10, problem 3.9]). Remember too that the dual problem for sections always has a positive answer. A star body $L$ in $\mathbb{R}^{n}$ having the origin in its interior is said to be of constant $i$-section if the $i$-volume of $L \cap S$ is the same for every $i$-dimensional subspace $S$ of $\mathbb{R}^{n}$. Gardner and Volčič showed ([11]; [10, 7.2.16]) that every star body in $\mathbb{R}^{n}$, having the origin in its interior, of constant $i$-section and $j$-section $(1 \leq i<j<n)$ is a centered ball.

A more general question can be formulated ([10, problem 3.10]; [12]):

Let $K$ and $L$ be two convex bodies in $\mathbb{R}^{n}$, $L$ being centrally symmetric. Are $K$ and $L$ homothetic if the ratio of the volumes of their projections on any i-dimensional subspace is, for two distinct values of $i(1 \leq i<n)$, a constant (depending on $i)$ ?

When $n=3, i=1,2$, Chakerian [5] proved that $K$ and $L$ are homothetic by imposing differentiability restrictions on the gauge body 
$L$ and the convex body $K$. This gave a generalisation of the Nakajima theorem to a 3-dimensional Minkowski space.

The following theorem answers the last question positively with some differentiability hypotheses:

Theorem B. Let two convex bodies $K, L$ in $\mathbb{R}^{n}$ be of class $C_{+}^{2}$, where $L$ is centrally symmetric. If, for two distinct values of $i(1 \leq i<n)$, the ratio of the volumes of their projections on any $i$-dimensional subspace is a constant (depending on $i$ ), then $K$ and $L$ are homothetic.

The notion of constant $k$-brightness implies that of constant $k$-girth (as defined in Section 2). In the final section, we observe that for a convex body $K$ in $\mathbb{R}^{n+1}$ with constant width, the continuous functions of $u \in \mathbb{S}^{n}$ which represent the $k$-girth in the direction $u$, satisfy linear dependence relationships. We thereby deduce:

Theorem C. Let $K$ be a convex body in $\mathbb{R}^{2 n}$ of class $C_{+}^{2}$ and constant width.

a) If $2 n \geq 6$ and $K$ has constant $(2 n-2)$-girth and constant $i_{j}$-girth for $n-2$ distinct integers $i_{j}$ with $2 \leq i_{j} \leq 2 n-3$, then $K$ is a ball.

b) If $n=2$ and $K$ has constant 2-girth, then $K$ is a ball.

To finish, let us briefly explain the idea of the proof of Theorem A. It consists of three stages. First of all, the case $i=1, j=n-1$ must be treated separately. Then the following two theorems allow us to deal with the other cases. Let us recall that a point $x$ of a smooth $n$-dimensional manifold $M(n \geq 2)$ is called an umbilic point of an immersion $f: M \rightarrow \mathbb{R}^{n+1}$ if all the principal curvature of $f$ at $x$ are equal.

Theorem D. Let $i<j<n$ be integers and let $K$ be a convex body in $\mathbb{R}^{n}$ of class $C_{+}^{2}$ such that bd $K$ has a pair of umbilic points belonging to two parallel support planes. If $K$ has constant $i$-brightness and $j$ brightness, then $K$ is a ball.

Theorem D implies Theorem A if we admit the existence of umbilic points. Hypersurfaces of dimension $n>2$ in $\mathbb{R}^{n+1}$ do not generally have umbilic points. This is in contrast with closed surfaces immersed in $\mathbb{R}^{3}$ having non-zero Euler characteristic. In fact, if $M$ is a manifold of dimension $n>2$, then the set of immersions of $M$ in $\mathbb{R}^{n+1}$ contains a dense and open set of immersions without umbilic points ([8, 4.8 p.10]). However, we prove: 
Theorem E. Let $K$ be a convex body in $\mathbb{R}^{n}(n \geq 4)$ of class $C_{+}^{2}$. If $K$ has constant $k$-brightness for a certain integer $k$ where $1<k<n-2$ or if $K$ has constant $1-$ and $(n-2)$-brightness, the hypersurface bd $K$ has a least one pair of umbilic points which belong to two parallel support planes.

The proof of one of the important lemmas used in the proof of this last theorem was communicated to us by Jacques Boéchat. We wish to thank him for his contribution. We also thank R. J. Gardner, R. Schneider and J. Thévenaz who contributed to improving a preliminary version of this article.

\section{Definitions, notation and known results about convex bodies of constant $i$-brightness and $i$-girth}

We will denote by $B^{n}$ the unit ball in $\mathbb{R}^{n}$, by $\mathbb{S}^{n-1}$ the unit sphere in $\mathbb{R}^{n}$, by $\lambda_{k}$ the (outer) $k$-dimensional Hausdorff measure in $\mathbb{R}^{n}$, and by $\kappa_{n}$ the volume of $B^{n}$. We will denote by $u^{\perp}$ the subspace of $\mathbb{R}^{n}$ orthogonal to $u \in \mathbb{S}^{n-1}$ and by $E \mid S$ the orthogonal projection of the set $E$ onto the subspace $S$. All the projections we will consider will be orthogonal.

We will denote by $\mathcal{K}^{n}$ the space of convex bodies in $\mathbb{R}^{n}$, i.e., the compact convex subsets in $\mathbb{R}^{n}$ with non-empty interior. If $K \in \mathcal{K}^{n}$ is of class $C^{2}$, we will denote by $\nu:$ bd $K \rightarrow \mathbb{S}^{n-1}$ the Gauss map, by $I I$ the second fundamental form of bd $K$, by $k_{n}(x, t)$ the normal curvature at $x \in \mathrm{bd} K$ in the direction $t \in T_{x} \mathrm{bd} K$ and by $k_{i}(x)$ the principal curvatures of bd $K$ at $x$.

When $K$ is of class $C_{+}^{2}$, we will consider the reverse

$$
\nu^{-1}: \mathbb{S}^{n-1} \rightarrow \text { bd } K
$$

of the Gauss map, the reverse Weingarten map $([21,2.5])$

$$
D \nu^{-1}(u): T_{u} \mathbb{S}^{n-1} \rightarrow T_{u} \mathbb{S}^{n-1},
$$

$u \in \mathbb{S}^{n-1}$, the second reverse fundamental form of $K$ at $u$ defined by $\overline{I I}_{u}(v, w)=\left\langle D \nu^{-1}(u)(v), w\right\rangle$ and for all $t \in T_{u} \mathbb{S}^{n-1} \backslash\{0\}$,

$$
r(u, t)=\overline{I I}_{u}(t, t)<t, t>^{-1}
$$

the radius of curvature at $u$ in the direction $t$. The formula (1) is the analogue for $\overline{I I}_{u}$ of the normal curvature $k_{n}(x, t)$ of $I I_{x}$ at $x=\nu^{-1}(u)$ for 
the direction $t$. If $t$ is parallel to a principal direction $X_{i}(x)$ of $I I_{x}$, then $r\left(u, X_{i}(u)\right)$ is equal to $k_{n}^{-1}\left(x, X_{i}(x)\right)$ with $u=\nu(x)$. The eigenvalues of the reverse Gauss map are therefore equal to $r_{i}=r\left(u, X_{i}(u)\right)$. They are called principal radii of curvature of $K$ at $u$. We will denote by $S_{j}$, $j>0$, the $j$ th elementary symmetric function of the principal radii of curvature $r_{i}=k_{i}^{-1}(i=1, \ldots, n-1)$ :

$$
S_{j}=\sum_{1 \leq i_{1} \leq \ldots \leq i_{j} \leq n-1} r_{i_{1}} \cdots r_{i_{j}} \quad \text { where } j=1, \ldots, n-1 .
$$

The $j$ th normalised elementary symmetric function is defined to be

$$
s_{j}=\left(\begin{array}{c}
n-1 \\
j
\end{array}\right)^{-1} S_{j} .
$$

The notation $F_{K}^{(j)}$ is sometimes used (see [10]) in place of $s_{j}$. We use the notation $s_{j}^{K}$ when it is useful to indicate that it depends on the convex body $K$.

Let $r^{\uparrow}$ be the continuous map, defined by

$$
r^{\uparrow}(u)=\left(r_{1}(u), \ldots, r_{n-1}(u)\right)^{\uparrow}
$$

whose value at $u \in \mathbb{S}^{n-1}$ is the vector of principal radii of curvature of $K$ in increasing order. We denote the $i$ th component of $r^{\uparrow}(u)$ by $r_{i}^{\uparrow}(u)$.

A chord of a convex body $K$ is a normal (or a double normal) if it is perpendicular, at one (or two) of its extremities, to the support plane(s) of $K$.

We denote by $\mathcal{W}^{n}$ the class of all the convex bodies in $\mathcal{K}^{n}$ with constant width.

Lemma 2.1 ([10, 7.1.13]). If $K \in \mathcal{W}^{n}$, any normal of $K$ is a double normal.

In fact, a body $K \in \mathcal{K}^{n}$ has constant width if and only if every normal is a double normal $\left(\left[6\right.\right.$, p.54]). For all $K \in \mathcal{W}^{n}, u \in \mathbb{S}^{n-1}$, the chord $\left[\nu^{-1}(u), \nu^{-1}(-u)\right]$ is orthogonal to the support hyperplanes to $K$ at its extremities. Consequently, if $K \in \mathcal{W}^{n}$ has width $b$,

$$
\nu^{-1}(-u)=\nu^{-1}(u)-b u \text { and } D \nu^{-1}(u)+D \nu^{-1}(-u)=b I d .
$$

We deduce the following theorem from (2) and the above observations. 
Theorem 2.2. ([10, 3.3.19]; $\left[6\right.$, p.65]) Let $K \in \mathcal{W}^{n}$ be a convex body of class $C_{+}^{2}$ and width $b$. The principal radii of curvature of $K$ satisfy

$$
r_{i}^{\uparrow}(u)+r_{n-i}^{\uparrow}(-u)=b \quad \text { for each } u \in \mathbb{S}^{n-1} \text { and } 1 \leq i \leq n-1 .
$$

The $k$-girth of $K \in \mathcal{K}^{n}$ in direction $u \in \mathbb{S}^{n-1}$ is equal $([6$, p.81]) to the mixed volume $n V\left(K, \ldots, K, B^{n}, \ldots, B^{n},[u]\right)$ where $K$ appears $k$ times, $B^{n}$ appears $(n-k-1)$ times and where $[u]$ is a line segment of unit length parallel to $u$. A convex body $K \in \mathcal{K}^{n}$ has constant $k$-girth if its $k$-girth is the same for any direction $u \in \mathbb{S}^{n-1}$. If $k=n-2, K$ is simply said to have constant girth, because the $(n-2)$-girth of $K$ in direction $u$ is equal to $\frac{1}{2} \lambda_{n-2}\left(\operatorname{bd}\left(K \mid u^{\perp}\right)\right)([10$, p.110]).

Theorem 2.3 ([9]; [10, 3.3.12-13]). a) (Firey) Every convex body $K \in \mathcal{K}^{n}$ with constant $k$-brightness where $1 \leq k<n$ also has constant $k$-girth.

b) (Minkowski, 1904, for $n=3$ ) If $k=1$, a convex body $K \in \mathcal{K}^{n}$ has constant width if and only if it has constant 1-girth.

c) If $k=n-1$ or $K$ is a convex body of revolution, then constant $k$-brightness and constant $k$-girth are equivalent.

Theorem 2.4. a) ([10, 3.3.14]) $A$ convex body $K \in \mathcal{K}^{n}$ of class $C_{+}^{2}$ has constant $k$-girth if and only if $s_{k}(u)+s_{k}(-u)$ is constant for all $u \in \mathbb{S}^{n-1}$.

b) ([9]) A convex body $K \in \mathcal{K}^{n}$ of class $C_{+}^{2}$ has constant $k$-brightness if and only if

$$
s_{k}(u)+s_{k}(-u)=2 \kappa_{k}^{-1} \lambda_{k}(K \mid E)
$$

for each $k$-dimensional subspace $E \subset \mathbb{R}^{n}$.

Recall that $G(n, k)$ denotes the Grassmann manifold of $k$-dimensional subspaces of $\mathbb{R}^{n}$.

Proposition 2.5 ([10,3.1.6]). Let $K \in \mathcal{K}^{n}$ and let $k$ be an integer where $2 \leq k \leq n-1$. If every projection $K \mid E, E \in G(n, k)$ is a $k$-dimensional ball, then $K$ is a $n$-dimensional ball.

Theorem 2.6 ([10,9.3.3]). Let $1 \leq k \leq n-1$, and suppose that $K \in \mathcal{K}^{n}$ and that $L$ is a zonoid in $\mathbb{R}^{n}$. Then

$$
\left(\frac{\lambda_{n}(L)}{\lambda_{n}(K)}\right)^{k / n} \geq \min _{E \in G(n, k)} \frac{\lambda_{k}(L \mid E)}{\lambda_{k}(K \mid E)},
$$

with equality if and only if $K$ and $L$ are homothetic. 


\section{Proof of Theorem D}

Let $a=\left(a_{1}, \ldots, a_{n}\right)$ be an $n$-tuple of positive real numbers and let $r$ be an integer where $1 \leq r \leq n$. The power mean of order $r$ of $a$ is defined by

$$
\mathcal{M}_{r}=\left(\frac{1}{n} \sum_{i=1}^{n} a_{i}^{r}\right)^{1 / r}
$$

It satisfies Jensen's inequality $([16,3.1 .1])$

$$
\mathcal{M}_{r} \leq \mathcal{M}_{s} \quad \text { if } r<s,
$$

with equality if and only if $a_{1}=\cdots=a_{n}$.

Theorem D. Let $i<j<n$ be integers and let $K$ be a convex body in $\mathbb{R}^{n}$ of class $C_{+}^{2}$ such that bd $K$ has a pair of umbilic points belonging to two parallel support planes. If $K$ has constant $i$-brightness and $j$ brightness, then $K$ is a ball.

Proof. By hypothesis the hypersurface bd $K$ has a pair of umbilic points belonging to two parallel support hyperplanes. Choose $u_{0} \in \mathbb{S}^{n-1}$ in such a way that $\nu^{-1}\left(u_{0}\right)$ and $\nu^{-1}\left(-u_{0}\right)$ are umbilic points. We will denote their radii of curvature by $x$ and $y$. Suppose that $K$ has constant $j$-brightness. We have by $(4), s_{j}(u)+s_{j}(-u)=2 \kappa_{j}^{-1} \lambda_{j}(K \mid E)$ for all $E \in G(n, j), u \in \mathbb{S}^{n-1}$. Consequently,

$$
\frac{\lambda_{j}(K \mid E)}{\kappa_{j}}=\frac{1}{2}\left(s_{j}\left(u_{0}\right)+s_{j}\left(-u_{0}\right)\right)=\frac{1}{2}\left(x^{j}+y^{j}\right) .
$$

If $K$ has constant $i$-brightness, we have for all $F \in G(n, i)$,

$$
\frac{\lambda_{i}(K \mid F)}{\kappa_{i}}=\frac{1}{2}\left(s_{i}\left(u_{0}\right)+s_{i}\left(-u_{0}\right)\right)=\frac{1}{2}\left(x^{i}+y^{i}\right) .
$$

As $i<j$, we have by Jensen's inequality:

$$
\left(\frac{\lambda_{i}(K \mid F)}{\kappa_{i}}\right)^{1 / i}=\left(\frac{1}{2}\left(x^{i}+y^{i}\right)\right)^{1 / i} \leq\left(\frac{1}{2}\left(x^{j}+y^{j}\right)\right)^{1 / j}=\left(\frac{\lambda_{j}(K \mid E)}{\kappa_{j}}\right)^{1 / j} .
$$

Suppose that $E \in G(n, j)$. We observe that $K \mid E$ has constant $i$ brightness. If $L$ denotes the unit ball in $\mathbb{R}^{j}=E$, we have $\kappa_{j}=\lambda_{j}(L)$ and $\kappa_{i}=\lambda_{i}(L \mid F)$ for all $F \in G(n, i)$. Theorem 2.6 applied to $K \mid E$ 
and $L$ asserts that the following inequalities are equalities if and only if $K \mid E$ is a ball:

$$
\left(\frac{\kappa_{j}}{\lambda_{j}(K \mid E)}\right)^{i / j} \geq \frac{\kappa_{i}}{\lambda_{i}(K \mid F)}, \quad \text { i.e., } \quad\left(\frac{\lambda_{i}(K \mid F)}{\kappa_{i}}\right)^{1 / i} \geq\left(\frac{\lambda_{j}(K \mid E)}{\kappa_{j}}\right)^{1 / j} \text {. }
$$

The reverse inequality derived above implies that the latter inequality is in fact an equality. Consequently $K \mid E$ is a ball for all $E \in G(n, j)$. We conclude that $K$ is an $n$-dimensional ball by Proposition 2.5. q.e.d.

\section{Characterisation of convex bodies of constant k-brightness}

In order to guarantee the existence of umbilic points on the hypersurfaces we will consider, we will generalize the criterion of Theorem 2.4 b) which characterizes convex bodies with constant $k$-brightness.

Let $Q_{k, n}$ be the set of all strictly increasing $k$-tuples of integers belonging to the set $\{1, \ldots, n\}$. The multi-index $\mathcal{I}$ belongs to $Q_{k, n}$ if and only if $\mathcal{I}=\left(i_{1}, \ldots, i_{k}\right)$ where $1 \leq i_{1}<i_{2}<\cdots<i_{k} \leq n$. Let $\left(e_{1}, \ldots, e_{n}\right)$ be an orthonormal basis of a Euclidean space $H$. For all $\mathcal{I} \in Q_{k, n}$, let $e_{\mathcal{I}}=e_{i_{1}} \wedge \ldots \wedge e_{i_{k}}$. The family $\left(e_{\mathcal{I}}\right)_{\mathcal{I} \in Q_{k, n}}$ is an orthonormal basis of the Euclidean space $\wedge^{k} H$.

Suppose that $\mathcal{I}, \mathcal{J} \in Q_{k, n}$. If, relative to the basis $\left(e_{1}, \ldots, e_{n}\right)$ of $H$, the endomorphism $A$ is represented by the matrix $\left(a_{i j}\right)$, we will denote by $A[\mathcal{I} \mid \mathcal{J}]$ the square sub-matrix of order $k$ of $A$ equal to $\left(a_{i j}\right)$ with $i \in \mathcal{I}, j \in \mathcal{J}$. The coefficient $(\mathcal{I}, \mathcal{J})$ of $\wedge^{k} A$ relative to the basis $\left(e_{\mathcal{I}}\right)_{\mathcal{I} \in Q_{k, n}}$ is equal to $\left(\wedge^{k} A\right)_{\mathcal{I} \mathcal{J}}=<e_{\mathcal{I}}, \wedge^{k} A e_{\mathcal{J}}>=\operatorname{det}(A[\mathcal{I} \mid \mathcal{J}])$.

Theorem 4.1. Let $K \in \mathcal{K}^{n}$ be a convex body of class $C_{+}^{2}$ and let $k$ be an integer with $1 \leq k<n$. Then $K$ has constant $k$-brightness if and only if

$$
\wedge^{k} D \nu^{-1}(u)+\wedge^{k} D \nu^{-1}(-u)=2 \kappa_{k}^{-1} \lambda_{k}(K \mid E) \wedge^{k} I d, \quad \forall u \in \mathbb{S}^{n-1},
$$

$D \nu^{-1}(u): T_{u} \mathbb{S}^{n-1} \rightarrow T_{u} \mathbb{S}^{n-1}$ denoting the reverse Weingarten map of

$K$ at $u$. Thus, for all $u \in \mathbb{S}^{n-1}, \wedge^{k} D \nu^{-1}(u)+\wedge^{k} D \nu^{-1}(-u)$ is a scalar multiple of the identity of $\wedge^{k} T_{u} \mathbb{S}^{n-1}$.

Proof. Suppose that $K$ has constant $k$-brightness. For all $E \in$ $G(k+1, n)$, the convex body $K \mid E$ has constant $(k-)$ brightness. Let $u \in \mathbb{S}^{n-1} \cap E$ and let

$$
\pi: T_{u} \mathrm{bd} K \rightarrow T_{u} \mathrm{bd}(K \mid E) \quad \text { and } \quad \eta: T_{u} \mathrm{bd}(K \mid E) \rightarrow T_{u} \mathrm{bd} K
$$


be respectively the orthogonal projection and the canonical inclusion. The second reverse fundamental form of $K \mid E$ at $u$ is equal, for all $v, w \in T_{u} \mathrm{bd}(K \mid E)$, to

$$
\overline{I I}_{u}^{K \mid E}(v, w)=<D \nu^{-1}(u) v, w>=<\pi D \nu^{-1}(u) \eta(v), w>.
$$

We denote by $\rho_{i}(u)$ the principal radii of curvature of the second reverse fundamental form of $K \mid E$ at $u$, and by $s_{k}^{K \mid E}(u)=\prod_{i=1}^{k} \rho_{i}(u)$ the $k$ th elementary symmetric function of the principal radii of curvature of $K \mid E$ at $u$.

Let $\left(w_{1}, \ldots, w_{k}\right)$ be an orthonormal basis of $T_{u} \mathrm{bd}(K \mid E)$, and $\left(u_{1}, \ldots, u_{n-1}\right)$ be an (orthonormal) basis of $T_{u} \mathrm{bd} K$ consisting of eigenvectors of $D \nu^{-1}(u)$. The vector $w=w_{1} \wedge \ldots \wedge w_{k} \in \wedge^{k} T_{u}$ bd $(K \mid E) \subset$ $\wedge^{k} T_{u}$ bd $K$ can be written as a linear combination of the vectors in the orthonormal basis $\left(u_{\mathcal{I}}\right)_{\mathcal{I} \in Q_{k, n-1}}$ of $\wedge^{k}\left(T_{u} \mathrm{bd} K\right)$, say $w=\sum_{\mathcal{I} \in Q_{k, n-1}} \alpha_{\mathcal{I}} u_{\mathcal{I}}$ where $\alpha_{\mathcal{I}}=<w, u_{\mathcal{I}}>$. Consequently, by ([16, I.4.6 (3)]),

$$
\begin{aligned}
& <w, \wedge^{k} D \nu^{-1}(u) w>=\sum_{\mathcal{J} \in Q_{k, n-1}}<w, \wedge^{k} D \nu^{-1}(u) u_{\mathcal{J}}><w, u_{\mathcal{J}}> \\
= & \sum_{\mathcal{J} \in Q_{k, n-1}} \operatorname{det}\left(\left(<w_{i}, D \nu^{-1}(u) u_{j}>\right)_{\substack{j \in \mathcal{J} \\
i=1, \ldots, k}}\right) \operatorname{det}\left(\left(<w_{i}, u_{j}>\right)_{\substack{j \in \mathcal{J} \\
i=1, \ldots, k}}\right) .
\end{aligned}
$$

The coefficients of the matrix $A \in M_{n-1, k}(\mathbb{R})$ of the inclusion $\eta$ relative to the pair of ordered bases $\left(w_{1}, \ldots, w_{k}\right)$ and $\left(u_{1}, \ldots, u_{n-1}\right)$ are equal to $a_{j i}=\left\langle w_{i}, u_{j}\right\rangle$. Those of the matrix $B \in M_{k, n-1}(\mathbb{R})$ of $\pi D \nu^{-1}(u)$ relative to the same bases are equal to

$$
b_{i j}=<w_{i}, D \nu^{-1}(u) u_{j}>\text {. }
$$

Suppose that $\mathcal{I}_{0}=(1, \ldots, k) \in Q_{k, k}$; then the former expression, by the Binet-Cauchy formula ([16, I.2.4.14]), is equal to

$$
\begin{aligned}
\sum_{\mathcal{J} \in Q_{k, n-1}} \operatorname{det}\left(B\left[\mathcal{I}_{0} \mid \mathcal{J}\right]\right) \operatorname{det}\left(A\left[\mathcal{J} \mid \mathcal{I}_{0}\right]\right) \\
=\operatorname{det}(B A)\left[\mathcal{I}_{0} \mid \mathcal{I}_{0}\right] \\
=\operatorname{det}(B A) \\
=\prod_{i=1}^{k} \rho_{i}(u)=s_{k}^{K \mid E}(u) .
\end{aligned}
$$

We denote by $T$ the matrix of $D \nu^{-1}(u)$ (or $D \nu^{-1}(-u)$ ) relative to the orthonormal basis $\left(u_{1}, \ldots, u_{n-1}\right)$. As $D \nu^{-1}(u)$ and $D \nu^{-1}(-u)$ are 
self-adjoint linear automorphisms of $T_{u}$ bd $K$, we have

$$
\begin{aligned}
<u_{\mathcal{I}}, \wedge^{k} T u_{\mathcal{J}}> & =\operatorname{det}(T[\mathcal{I} \mid \mathcal{J}]) \\
& =\operatorname{det}\left(T^{t}[\mathcal{J} \mid \mathcal{I}]\right) \\
& =\operatorname{det}(T[\mathcal{J} \mid \mathcal{I}]) \\
& =<u_{\mathcal{J}}, \wedge^{k} T u_{\mathcal{I}}>
\end{aligned}
$$

Thus $\wedge^{k} D \nu^{-1}(u)$ and $\wedge^{k} D \nu^{-1}(-u)$ are self-adjoint linear automorphisms of $\wedge^{k}\left(T_{u}\right.$ bd $\left.K\right)$. The function $Q: \wedge^{k}\left(T_{u}\right.$ bd $\left.K\right) \rightarrow \mathbb{R}$ defined by

$$
Q(w)=<w,\left(\wedge^{k} D \nu^{-1}(u)+\wedge^{k} D \nu^{-1}(-u)\right) w>
$$

is therefore a quadratic form on $\wedge^{k}\left(T_{u}\right.$ bd $\left.K\right)$.

Let $E \in G(k+1, n)$ such that $u \in \mathbb{S}^{n-1} \cap E$, let $\left(w_{i}\right)_{i=1}^{k}$ be an orthonormal basis of the subspace of $E$ orthogonal to $u$ and let $w=$ $w_{1} \wedge \cdots \wedge w_{k}$ be the elementary symmetric tensor of norm one. In view of (5), the value of the quadratic form $Q$ at $w$ is equal to

$$
s_{k}^{K \mid E}(u)+s_{k}^{K \mid E}(-u)=2 \kappa_{k}^{-1} \lambda_{k}(K \mid E) .
$$

This number is independent of $w$ by Theorem $2.4 \mathrm{~b}$ ). In particular $Q$ takes the same value on each of the vectors $u_{\mathcal{I}}$, which constitute an orthonormal basis of $\wedge^{k}\left(T_{u}\right.$ bd $\left.K\right)$. The quadratic form $Q$ is therefore constant on the unit sphere of $\wedge^{k}\left(T_{u} \mathrm{bd} K\right)$. The self-adjoint endomorphism $\wedge^{k} D \nu^{-1}(u)+\wedge^{k} D \nu^{-1}(-u)$ associated to $Q$ is thus a scalar multiple of the identity.

The converse follows from Theorem $2.4 \mathrm{~b}$ ) and the fact that $\operatorname{tr} \wedge^{k} A$ is the $k$ th-elementary symmetric function of the eigenvalues of $A$. q.e.d.

Corollary 4.2. Let $K \in \mathcal{K}^{n}$ be a convex body of class $C_{+}^{2}$ and let $k$ be an integer with $1 \leq k<n$. If the convex body $K$ has constant $k$ brightness, then the reverse Weingarten maps $D \nu^{-1}(u)$ and $D \nu^{-1}(-u)$ are simultaneously orthogonally diagonalisable.

\section{Schur-convex functions and elementary symmetric functions}

Let us introduce the notion of a Schur-convex function. This will enable us to characterize balls in $\mathbb{R}^{n}$ as convex bodies with constant width and $(n-1)$-brightness without needing to establish the existence 
of umbilic points. In the second half of the section, from Lemma 5.4 onwards, we establish the lemmas needed in the following section in order to prove the existence of umbilic points on $(n-1)$-dimensional hypersurfaces with constant $k$-brightness for $k<n-1$.

Let $\lambda=\left(\lambda_{1}, \ldots, \lambda_{n}\right) \in \mathbb{R}^{n}$. We will denote by $\lambda^{\uparrow}$ (or $\lambda^{\downarrow}$ ) the vector of $\mathbb{R}^{n}$ obtained by reordering the components of $\lambda$ in increasing (or decreasing) order. We will denote by $\lambda[i]$ (or $\lambda[i, j]$ ) the vector of $\mathbb{R}^{n-1}$ (or $\mathbb{R}^{n-2}$ ) obtained from $\lambda$ by omitting its $i$ th (or $i$ th and $j$ th) component(s).

If $x, y \in \mathbb{R}^{n}$, we will say that $y$ majorizes $x$ and will use the symbol

$$
x \prec y \quad \text { if } \quad\left\{\begin{array}{l}
\sum_{i=1}^{k} x_{i}^{\downarrow} \leq \sum_{i=1}^{k} y_{i}^{\downarrow} \quad k=1, \ldots, n-1, \\
\sum_{i=1}^{n} x_{i}^{\downarrow}=\sum_{i=1}^{n} y_{i}^{\downarrow} .
\end{array}\right.
$$

More generally, $x$ is said to be weakly supermajorized by $y$, written

$$
x \prec w y \quad \text { if } \quad \sum_{i=1}^{k} x_{i}^{\uparrow} \leq \sum_{i=1}^{k} y_{i}^{\uparrow} \quad k=1, \ldots, n .
$$

Suppose that $A \subset \mathbb{R}^{n}$. We will write $x \prec y$ on $A$ if $x, y \in A$ and $x \prec y$. A real function $\phi$ defined on $A$ is said to be $S$ chur-convex on $A$ if $x \prec y$ on $A$ implies $\phi(x) \leq \phi(y)$. It is strictly Schur-convex on $A$ if we have $\phi(x)<\phi(y)$ when $x \prec y$ on $A$ and if $x$ is not a permutation of $y$. Finally the function $\phi$ is said to be (strictly) Schur-concave on $A$ if $-\phi$ is (strictly) Schur-convex on $A$.

Theorem 5.1 ([17, 3.A.4]). Let $I \subset \mathbb{R}$ be an open interval and let $\phi: I^{n} \rightarrow \mathbb{R}$ be a continuously differentiable function. The function $\phi$ is Schur-convex on $I^{n}$ if and only if it is symmetric on $I^{n}$ (i.e., $\phi(x)=$ $\phi(\sigma(x))$ for all $x \in I^{n}$ and all permutations $\sigma$ ) and if for all $i \neq j$,

$$
\left(x_{i}-x_{j}\right)\left[\frac{\partial}{\partial x_{i}} \phi(x)-\frac{\partial}{\partial x_{j}} \phi(x)\right] \geq 0 \quad \text { for all } x \in I^{n} .
$$

Condition (6) is called Schur's condition. If the inequality (6) is strict for all $x \in I^{n}$ where $x_{i} \neq x_{j}$, the function $\phi$ is strictly Schurconvex on $I^{n}$; see [20, Theorem VIII].

Theorem 5.2 ([17, 3.A.8.a]). Let $I \subset \mathbb{R}$ be an open interval and let $\phi: I^{n} \rightarrow \mathbb{R}$ be a continuously differentiable function. The function $\phi$ is strictly decreasing and strictly Schur-convex on $I^{n}$ if and only if the following condition holds:

whenever $x \prec^{w} y$ on $I^{n}$ and $x$ is not a permutation of $y$, then $\phi(x)<$ $\phi(y)$. 
We will introduce some more notation.

Let $\mathbb{R}_{+}^{n}=\left\{x \in \mathbb{R}^{n}: x_{i} \geq 0, \forall i\right\}$ and $\mathbb{R}_{++}^{n}=\left\{x \in \mathbb{R}^{n}: x_{i}>0, \forall i\right\}$. For all $a \in \mathbb{R}$, let $\mathbf{a}=(a, \ldots, a) \in \mathbb{R}^{n}$. If $x=\left(x_{1}, \ldots, x_{n}\right) \in \mathbb{R}^{n}, \mathbf{a}-x$ is the vector with components $\left(a-x_{1}, \ldots, a-x_{n}\right)$.

If $k \in \mathbb{N}$, the function $S_{k}: \mathbb{R}^{n} \rightarrow \mathbb{R}$ defined by

$$
S_{k}(x)=\sum_{1 \leq i_{1}<\cdots<i_{k} \leq n} x_{i_{1}} \cdots x_{i_{k}}
$$

is called the $k$ th elementary symmetric polynomial of the $n$ variables $x_{1}, \ldots, x_{n}$. The identities

$$
\begin{aligned}
& \frac{\partial}{\partial x_{j}} S_{k}(x)=S_{k-1}(x[j]), \\
& S_{k}(x[i])-S_{k}(x[j])=\left(x_{j}-x_{i}\right) S_{k-1}(x[i, j])
\end{aligned}
$$

easily imply $([17,3 . \mathrm{F} .1])$ that the function $S_{k}$ is Schur-concave on $\mathbb{R}_{+}^{n}$ and that $S_{k}$ is strictly Schur-concave on $\mathbb{R}_{++}^{n}$ if $k>1$.

Lemma 5.3. For all $x \in \mathbb{R}^{n}$, let $\tilde{x}=\mathbf{1}-x$. The function $F$ : $(0,1)^{n} \rightarrow \mathbb{R}$ defined by

$$
F(x)=S_{n}(x)+S_{n}(\tilde{x})=S_{n}(x)+\sum_{i=0}^{n}(-1)^{i} S_{i}(x)
$$

is, for $n>1$, strictly increasing on $(0,1)^{n}$ and strictly Schur-concave.

Proof. In view of $([17,16$ A.1.a $])$, it is enough to verify that $\nabla F(x)>0$ in order for $F$ to be strictly increasing. This condition is satisfied, because, by (7), we have

$\frac{\partial F}{\partial x_{j}}(x)=S_{n-1}(x[j])+\sum_{i=1}^{n}(-1)^{i} S_{i-1}(x[j])=S_{n-1}(x[j])+S_{n-1}(\tilde{x}[j])>0$.

To conclude, one verifies Schur's condition (using the identities (7) between the elementary symmetric polynomials). q.e.d.

Now we will prove some lemmas which determine the solutions of equations in which elementary symmetric functions appear.

Recall that, by definition, $S_{-1}(\lambda)=0$ and $S_{0}(\lambda)=1$ and that

$$
S_{k}(\lambda)=S_{k}(\lambda[i])+\lambda_{i} \cdot S_{k-1}(\lambda[i]) \quad \forall \lambda \in \mathbb{R}^{n} .
$$

We are grateful to Jacques Boéchat for having communicated to us the proof of the following lemma: 
Lemma 5.4. Let $\lambda=\left(\lambda_{1}, \ldots, \lambda_{n}\right) \in \mathbb{R}^{n}$ where $n \geq 3$ and let $1 \leq i<p<j \leq n$ be integers. If there are two integers $1 \leq k<\ell<n$ such that

$$
S_{k}(\lambda[i])+S_{k}(\lambda[j])=2 S_{k}(\lambda[p]) \text { and } S_{\ell}(\lambda[i])+S_{\ell}(\lambda[j])=2 S_{\ell}(\lambda[p])
$$

then $\lambda_{i}=\lambda_{j}=\lambda_{p}$.

Proof. For simplicity, we write $\check{S}_{k}=S_{k}(\lambda[i, p, j])$. We will apply (8) successively to $S_{k}(\lambda[i]), S_{k}(\lambda[p])$ and $S_{k}(\lambda[j])$. We obtain

$$
S_{k}(\lambda[i])=\check{S}_{k}+\left(\lambda_{p}+\lambda_{j}\right) \check{S}_{k-1}+\lambda_{p} \lambda_{j} \check{S}_{k-2}
$$

and, after permuting the indices, similar expressions for $S_{k}(\lambda[p])$ and $S_{k}(\lambda[j])$. The first equality in the hypothesis is now written as

$$
\begin{aligned}
2 \breve{S}_{k}+\left(\lambda_{i}+2 \lambda_{p}+\lambda_{j}\right) \check{S}_{k-1}+\lambda_{p}\left(\lambda_{i}+\lambda_{j}\right) \check{S}_{k-2} \\
=2\left(\breve{S}_{k}+\left(\lambda_{i}+\lambda_{j}\right) \breve{S}_{k-1}+\lambda_{i} \lambda_{j} \breve{S}_{k-2}\right)
\end{aligned}
$$

or

$$
2 \lambda_{p} \breve{S}_{k-1}+\left(\lambda_{i}+\lambda_{j}\right)\left[\lambda_{p} \breve{S}_{k-2}-\breve{S}_{k-1}\right]-2 \lambda_{i} \lambda_{j} \breve{S}_{k-2}=0 .
$$

The second equality gives the same relationship except that $k$ is replaced by $\ell$. That is to say, $\left(\lambda_{i}+\lambda_{j}, \lambda_{i} \lambda_{j}\right)$ is a solution of the following system of linear equations in $(X, Y)$ :

$$
\left(\begin{array}{cc}
\check{S}_{k-1}-\lambda_{p} \check{S}_{k-2} & 2 \check{S}_{k-2} \\
\check{S}_{\ell-1}-\lambda_{p} \check{S}_{\ell-2} & 2 \check{S}_{\ell-2}
\end{array}\right)\left(\begin{array}{c}
X \\
Y
\end{array}\right)=2 \lambda_{p}\left(\begin{array}{c}
\check{S}_{k-1} \\
\check{S}_{\ell-1}
\end{array}\right)
$$

However $\left(2 \lambda_{p}, \lambda_{p}^{2}\right)$ is also a solution of $(9)$. To arrive at a conclusion, we only have to prove that (9) has a unique solution, i.e., that

$$
\left(\check{S}_{k-1} \check{S}_{\ell-2}-\check{S}_{\ell-1} \check{S}_{k-2}\right) \neq 0
$$

We claim that $\check{S}_{k-2} / \check{S}_{k-1}<\check{S}_{\ell-2} / \check{S}_{\ell-1}$.

For all fixed $\lambda \in \mathbb{R}^{m}$, we recall that the $k$ th normalised elementary symmetric function is written

$$
s_{k}=s_{k}(\lambda)=\left(\begin{array}{c}
m \\
k
\end{array}\right)^{-1} S_{k}(\lambda) \quad(k=0, \ldots, m)
$$


The inequalities $\frac{s_{k-1}}{s_{k}} \leq \frac{s_{k}}{s_{k+1}}$, known as Newton inequalities ([16, II.3.2.1]), between the successive ratios of $s_{k}$ for $k=0, \ldots, m-1$ and the inequalities $\left(\begin{array}{c}m \\ k-1\end{array}\right)\left(\begin{array}{c}m \\ k\end{array}\right)^{-1}<\left(\begin{array}{c}m \\ k\end{array}\right)\left(\begin{array}{c}m \\ k+1\end{array}\right)^{-1}$ enable us to prove

$$
\frac{S_{k-1}(\lambda)}{S_{k}(\lambda)}=\left(\begin{array}{c}
m \\
k-1
\end{array}\right)\left(\begin{array}{c}
m \\
k
\end{array}\right)^{-1} \frac{s_{k-1}}{s_{k}}<\left(\begin{array}{c}
m \\
k
\end{array}\right)\left(\begin{array}{c}
m \\
k+1
\end{array}\right)^{-1} \frac{s_{k}}{s_{k+1}}=\frac{S_{k}(\lambda)}{S_{k+1}(\lambda)} .
$$

This proves the claim. q.e.d.

Lemma 5.5. Let $\lambda=\left(\lambda_{1}, \ldots, \lambda_{n}\right) \in \mathbb{R}^{n}$ where $n \geq 4$ and let $1 \leq i, p, q, j \leq n$ be distinct integers. If an integer $1<k<n$ exists such that

$$
\begin{gathered}
S_{k}(\lambda[i, p])+S_{k}(\lambda[q, j])=2 S_{k}(\lambda[p, q]) \text { and } \\
S_{k}(\lambda[j, p])+S_{k}(\lambda[q, i])=2 S_{k}(\lambda[p, q]),
\end{gathered}
$$

then $\lambda_{i}=\lambda_{j}$ if $\lambda_{p} \neq \lambda_{q}$.

Proof. For simplicity, write $\check{S}_{k}=S_{k}(\lambda[i, p, q, j])$. By applying (8) successively to $S_{k}(\lambda[i, p]), S_{k}(\lambda[q, j])$ and $S_{k}(\lambda[p, q])$, we obtain

$$
\begin{aligned}
& S_{k}(\lambda[i, p])=\check{S}_{k}+\left(\lambda_{j}+\lambda_{q}\right) \check{S}_{k-1}+\lambda_{j} \lambda_{q} \check{S}_{k-2}, \\
& S_{k}(\lambda[q, j])=\check{S}_{k}+\left(\lambda_{i}+\lambda_{p}\right) \check{S}_{k-1}+\lambda_{i} \lambda_{p} \check{S}_{k-2}, \\
& S_{k}(\lambda[p, q])=\check{S}_{k}+\left(\lambda_{i}+\lambda_{j}\right) \check{S}_{k-1}+\lambda_{i} \lambda_{j} \check{S}_{k-2} .
\end{aligned}
$$

After having made the above substitutions, the first of the equations (10) becomes

$$
\left(\lambda_{p}+\lambda_{q}\right) \check{S}_{k-1}+\left(\lambda_{j} \lambda_{p}+\lambda_{i} \lambda_{q}\right) \check{S}_{k-2}=\left(\lambda_{i}+\lambda_{j}\right) \check{S}_{k-1}+2 \lambda_{i} \lambda_{j} \check{S}_{k-2} .
$$

In order to move from one equation to the other in (10), one only has to exchange the roles of $i$ and $j$. Thus $\left(\lambda_{j} \lambda_{p}+\lambda_{i} \lambda_{q}\right)=\left(\lambda_{i} \lambda_{p}+\lambda_{j} \lambda_{q}\right)$, i.e., $\lambda_{j}\left(\lambda_{p}-\lambda_{q}\right)=\lambda_{i}\left(\lambda_{p}-\lambda_{q}\right)$. Consequently $\lambda_{i}=\lambda_{j}$ if $\lambda_{p} \neq \lambda_{q}$. q.e.d.

Lemma 5.6. Let $\lambda=\left(\lambda_{1}, \ldots, \lambda_{n}\right) \in \mathbb{R}^{n}$ where $n \geq 4$, and let $1 \leq i, p, q, j \leq n$ be distinct integers. If there are two integers $1 \leq k<\ell<n-1$ such that

$$
S_{k}(\lambda[i])+S_{k}(\lambda[j])=S_{k}\left(\lambda[p]+S_{k}(\lambda[q])\right.
$$

and

$$
S_{\ell}(\lambda[i])+S_{\ell}(\lambda[j])=S_{\ell}(\lambda[p])+S_{\ell}(\lambda[q]),
$$

then $\left(\lambda_{p}, \lambda_{q}\right)=\left(\lambda_{i}, \lambda_{j}\right)$ or $\left(\lambda_{j}, \lambda_{i}\right)$. 
Proof. For simplicity, write $\check{S}_{k}=S_{k}(\lambda[i, p, q, j])$. By applying (8) successively, we obtain that $S_{k}(\lambda[i])$ is equal to

$$
\check{S}_{k}+\left(\lambda_{j}+\lambda_{p}+\lambda_{q}\right) \check{S}_{k-1}+\left(\lambda_{p} \lambda_{q}+\lambda_{j} \lambda_{q}+\lambda_{p} \lambda_{j}\right) \check{S}_{k-2}+\lambda_{j} \lambda_{p} \lambda_{q} \check{S}_{k-3} .
$$

By permuting the indices, we obtain similar expressions for $S_{k}(\lambda[j])$, $S_{k}(\lambda[p])$ and $S_{k}(\lambda[q])$. Then (11) tell us that $\left(\lambda_{p}+\lambda_{q}, \lambda_{p} \lambda_{q}\right)$ is a solution of the following system of linear equations in $(X, Y)$ :

$$
\begin{gathered}
\left(\begin{array}{cc}
\check{S}_{k-1}-\lambda_{i} \lambda_{j} \check{S}_{k-3} & 2 \check{S}_{k-2}+\left(\lambda_{i}+\lambda_{j}\right) \check{S}_{k-3} \\
\check{S}_{\ell-1}-\lambda_{i} \lambda_{j} \check{S}_{\ell-3} & 2 \check{S}_{\ell-2}+\left(\lambda_{i}+\lambda_{j}\right) \check{S}_{\ell-3}
\end{array}\right)\left(\begin{array}{c}
X \\
Y
\end{array}\right) \\
=\left(\begin{array}{c}
\left(\lambda_{i}+\lambda_{j}\right) \check{S}_{k-1}+2 \lambda_{i} \lambda_{j} \check{S}_{k-2} \\
\left(\lambda_{i}+\lambda_{j}\right) \check{S}_{\ell-1}+2 \lambda_{i} \lambda_{j} \check{S}_{\ell-2}
\end{array}\right) .
\end{gathered}
$$

But $\left(\lambda_{i}+\lambda_{j}, \lambda_{i} \lambda_{j}\right)$ is also a solution of (12). In order to reach a conclusion, one only has to prove that (12) has a unique solution, i.e., that

$$
\begin{aligned}
\left(\check{S}_{k-1} \check{S}_{\ell-2}-\check{S}_{\ell-1} \check{S}_{k-2}\right) & +\left(\lambda_{i}+\lambda_{j}\right)\left(\check{S}_{k-1} \check{S}_{\ell-3}-\check{S}_{\ell-1} \check{S}_{k-3}\right) \\
& +\lambda_{i} \lambda_{j}\left(\check{S}_{k-2} \check{S}_{\ell-3}-\check{S}_{\ell-2} \check{S}_{k-3}\right) \neq 0 .
\end{aligned}
$$

This expression is non-zero by the claim established at the end of the proof of Lemma 5.4. q.e.d.

\section{Proof of Theorem E}

Proposition 6.1. Let $K \in \mathcal{W}^{n}$ be of class $C_{+}^{2}$ where $n \geq 4$. If $K$ has constant $(n-2)$-brightness, the hypersurface $\mathrm{bd} K$ has at least one pair of umbilic points which belong to two parallel support hyperplanes.

Proof. We shall use the notation $r^{\uparrow}(u)$ introduced before Lemma 2.1. By the Borsuk-Ulam theorem $([4,4.20 .2])$, there is a point $u_{0} \in$ $\mathbb{S}^{n-1}$ such that $r^{\uparrow}\left(u_{0}\right)=r^{\uparrow}\left(-u_{0}\right)$. In addition we know, by Theorem 2.2 , that if the width of $K$ is equal to $2 b$, then $r_{i}^{\uparrow}(u)+r_{n-i}^{\uparrow}(-u)=2 b$, $\forall u \in \mathbb{S}^{n-1}$.

Suppose that $n=2 m$ is even. Then $r_{m}^{\uparrow}\left(u_{0}\right)=b$. In addition, by Theorem 4.1, we have, for all $1 \leq i<n$,

$$
S_{k}\left(r^{\uparrow}\left(u_{0}\right)[i]\right)+S_{k}\left(r^{\uparrow}\left(u_{0}\right)[n-i]\right)=2 S_{k}\left(r^{\uparrow}\left(u_{0}\right)[m]\right), \quad k=1, n-2 .
$$

Consequently, $r_{1}^{\uparrow}\left(u_{0}\right)=r_{n-1}^{\uparrow}\left(u_{0}\right)=r_{m}^{\uparrow}\left(u_{0}\right)=b$ by Lemma 5.4. The points $\nu^{-1}\left( \pm u_{0}\right)$ are therefore the umbilic points we sought. 
Suppose that $n=2 m+1$ is odd. By Theorem 4.1, we have for all $1 \leq i, j<n$ and for $k=1$ or $n-2$

$$
S_{k}\left(r^{\uparrow}\left(u_{0}\right)[i]\right)+S_{k}\left(r^{\uparrow}\left(u_{0}\right)[n-i]\right)=S_{k}\left(r^{\uparrow}\left(u_{0}\right)[j]\right)+S_{k}\left(r^{\uparrow}\left(u_{0}\right)[n-j]\right) .
$$

Therefore, if we let $2 \varepsilon=r_{2 m}^{\uparrow}\left(u_{0}\right)-r_{1}^{\uparrow}\left(u_{0}\right)$, then by Lemma 5.6,

$$
r_{i}^{\uparrow}\left(u_{0}\right)=b-\varepsilon \quad \text { if } i \leq m \quad \text { and } \quad r_{i}^{\uparrow}\left(u_{0}\right)=b+\varepsilon \quad \text { if } i>m .
$$

Let $X_{i}(u) \in T_{\nu^{-1}(u)} \mathrm{bd} K$ be a principal direction corresponding to the eigenvalue $r_{i}^{\uparrow}(u)$ of $D \nu^{-1}(u)$. The convex bodies $K \mid X_{i}(u)^{\perp}$ have constant $(n-2)$-brightness. The following functions, by Theorem 2.4, are all equal to the same constant $C$, equal to $2 \kappa_{n-2}^{-1} \lambda_{n-2}(K \mid E), E \in G(n, k)$ :

$$
s_{n-2}^{K \mid X_{i}^{\perp}(u)}(v)+s_{n-2}^{K \mid X_{i}^{\perp}(u)}(-v)=C, \quad \forall v \in X_{i}(u)^{\perp} \cap \mathbb{S}^{n} .
$$

By $4.2, X_{i}(u)=X_{n-i}(-u)$. Thus these equations are equivalent to

$$
\prod_{j \neq i} r_{j}^{\uparrow}(u)+\prod_{j \neq n-i} r_{j}^{\uparrow}(-u)=\left(\begin{array}{c}
n-2 \\
n-2
\end{array}\right) C=C .
$$

Theorem $2.4 \mathrm{~b}$ ) implies that

$$
C=\left(\begin{array}{c}
n-1 \\
n-1
\end{array}\right) C=\prod_{j} r_{j}^{\uparrow}(u)+\prod_{j} r_{j}^{\uparrow}(-u) .
$$

By (13), the previous two equations with $u=u_{0}$ give

$$
2\left(b-\varepsilon^{2}\right)^{m}=C=2 b\left(b-\varepsilon^{2}\right)^{m-1} .
$$

Consequently $\varepsilon=0$. The points $\nu^{-1}\left( \pm u_{0}\right)$ are therefore the umbilic points we sought. q.e.d.

Proposition 6.2. Let $K \in \mathcal{K}^{n}$ be of class $C_{+}^{2}$ where $n \geq 4$ and let $k$ be an integer such that $1<k<n-2$. If $K$ has constant $k$ brightness, the hypersurface bd $K$ has at least one pair of umbilic points which belong to two parallel support hyperplanes.

Proof. As before (see Proposition 6.1 ), there is a point $u_{0} \in \mathbb{S}^{n-1}$ where $r^{\uparrow}\left(u_{0}\right)=r^{\uparrow}\left(-u_{0}\right)$. The convex bodies

$$
K_{i}=K \mid\left(X_{1}\left(u_{0}\right)^{\perp} \cap X_{i}\left(u_{0}\right)^{\perp}\right)
$$

have the same constant $k$-brightness for $i=2, \ldots, n-1$. Since

$$
\lambda_{k}\left(K_{2} \mid E\right)=\lambda_{k}\left(K_{n-1} \mid E\right)=\lambda_{k}\left(K_{n-2} \mid E\right)
$$


for any $E \in G(n, k)$, by applying Theorem 4.1 to $K_{i}$ for $i=2, n-2$ and $n-1$, we have, that

$$
S_{k}\left(r^{\uparrow}\left(u_{0}\right)[1,2]\right)+S_{k}\left(r^{\uparrow}\left(u_{0}\right)[n-2, n-1]\right)=2 S_{k}\left(r^{\uparrow}\left(u_{0}\right)[1, n-1]\right)
$$

and

$$
S_{k}\left(r^{\uparrow}\left(u_{0}\right)[1, n-2]\right)+S_{k}\left(r^{\uparrow}\left(u_{0}\right)[2, n-1]\right)=2 S_{k}\left(r^{\uparrow}\left(u_{0}\right)[1, n-1]\right) .
$$

Suppose that $D \nu^{-1}\left(u_{0}\right)$ is not an umbilic point. Then, by Lemma 5.5,

$$
\hat{r_{2}}\left(u_{0}\right)=r_{n-2}^{\uparrow}\left(u_{0}\right)
$$

because

$$
r_{1}^{\uparrow}\left(u_{0}\right) \neq r_{n-1}^{\uparrow}\left(u_{0}\right) .
$$

Choose an integer $i$ where $1<i<n-1$ and let $\varepsilon=r_{i}^{\uparrow}\left(u_{0}\right)$. As the components of $r^{\uparrow}\left(u_{0}\right)$ are in increasing order, $\varepsilon$ does not depend on the choice of $i$. As $k \leq n-3$, it follows from Theorem 4.1 that $2 \varepsilon^{k}=\left(r_{1}^{\uparrow}\left(u_{0}\right)+r_{n-1}^{\uparrow}\left(u_{0}\right)\right) \overline{\varepsilon^{k-1}}$ and that $2 \varepsilon^{k}=2 r_{1}^{\uparrow}\left(u_{0}\right) r_{n-1}^{\uparrow}\left(u_{0}\right) \varepsilon^{k-2}$. Consequently $r_{1}^{\uparrow}\left(u_{0}\right)=r_{n-1}^{\uparrow}\left(u_{0}\right)=\varepsilon$. Therefore $D \nu^{-1}\left(u_{0}\right)$ is an umbilic point, a contradiction.

Hence $D \nu^{-1}\left(u_{0}\right)$ must be an umbilic point. The point $D \nu^{-1}\left(-u_{0}\right)$ is also one by Theorem 4.1. q.e.d.

Theorem E follows directly from Propositions 6.1 and 6.2 .

\section{Proof of Theorem A}

Theorem 7.1. A convex body $K \in \mathcal{W}^{n}(n \geq 3)$ of class $C_{+}^{2}$ which has constant $(n-1)$-brightness is a ball.

Proof. Suppose that $b$ is the width of $K \in \mathcal{W}^{n}$. For all $u, v \in \mathbb{S}^{n-1}$,

$$
S_{n-1}\left(r^{\uparrow}(u)\right)+S_{n-1}\left(r^{\uparrow}(-u)\right)=S_{n-1}\left(r^{\uparrow}(v)\right)+S_{n-1}\left(r^{\uparrow}(-v)\right)
$$

by Theorem 2.4. Consequently, for all $u, v \in \mathbb{S}^{n-1}$, we have

$$
S_{n-1}\left(r^{\uparrow}(u)\right)+S_{n-1}\left(\mathbf{b}-r^{\uparrow}(u)\right)=S_{n-1}\left(r^{\uparrow}(v)\right)+S_{n-1}\left(\mathbf{b}-r^{\uparrow}(v)\right),
$$

because the principal radii of curvature of $K$ satisfy

$$
r_{n-i}^{\uparrow}(-u)=b-r_{i}^{\uparrow}(u), \quad \forall u \in \mathbb{S}^{n-1},
$$


(Theorem 2.2). Lemmas 5.2 and 5.3 enable us to claim that $r^{\uparrow}(u)=$ $r^{\uparrow}(v), \forall u, v \in \mathbb{S}^{n-1}$.

Thus $K$ is a ball (see [21, p. 301]), because the elementary symmetric functions of the principal radii of curvature are constant (or more easily because the Gauss-Kronecker curvature of $b d K$ is constant). q.e.d.

Theorem 7.2. A convex body $K \in \mathcal{W}^{n}$ of class $C_{+}^{2}$ which has constant $k$-brightness, where $1<k<n$, is a ball.

Proof. For all $E \in G(n, k+1)$ the convex body $K \mid E$ is of class $C_{+}^{2}$, and has constant width and $k$-brightness. Consequently, by Theorem $7.1, K \mid E$ is a ball for all $E \in G(n, k+1)$. We conclude that $K$ is an $n$-dimensional ball by Proposition 2.5 . q.e.d.

Theorem A. Let $0<i<j<n$ be integers and $K$ a convex body in $\mathbb{R}^{n}$ of class $C_{+}^{2}$. If $K$ has constant $i$-brightness and $j$-brightness, then $K$ is a ball.

Proof. If $(i, j)=(1, n-1)$, we know that the hypersurface bd $K$ is a sphere by Theorem 7.1.

If $(i, j) \neq(1, n-1)$, we have either $2 \leq i \leq n-3$, or $2 \leq j \leq n-3$, or $(i, j)=(1, n-2)$. Consequently the hypersurface bd $K$ has a pair of umbilic points belonging to two parallel support hyperplanes, either by Proposition 6.1 or by Proposition 6.2. Theorem D now enables us to complete the proof. q.e.d.

\section{Constant relative brightness}

In relative differential geometry in a finite dimensional vector space $X$, a given convex body $B \subset X$ (which is not necessarily centrally symmetric) is used as the "unit ball" in the geometry we are considering (cf $\left[3\right.$, p.64]). It is called the gauge body. We note that if $B \in \mathcal{K}^{n}$ is centrally symmetric, then $B$ can be considered as the unit ball in $X$. The space $X$ endowed with the associated norm is a Minkowski space denoted by $\left(X,\|\|_{B}\right)$ (see $\left.[23,1.1 .8]\right)$. The notions of constant Minkowski width, of Minkowski $k$-girth, etc., are obtained from those of relative geometry by taking the unit ball of the Minkowski space as the gauge body.

Numerous authors have studied sets of constant width in a Minkowski space, i.e., in a finite-dimensional normed space. We can quote Chakerian and Groemer's survey [6] and that of Heil and Martini [14], as well as Thompson's book ([23, $\$ 4.2$ and 4.8]) and that of Boltyanski, Martini 
and Soltan $([2, \S 32])$. Obviously there are several characterisations of convex bodies of constant width in a Minkowski space $\left(X,\|\|_{B}\right)$ with unit ball $B$.

Let $B \in \mathcal{K}^{n}$ be a gauge body of class $C_{+}^{2}$ and let $K \in \mathcal{K}^{n}$ be a convex body of class $C_{+}^{2}$. We refer to Leichtweiss $([15, \S 6$, pp.1075-6]) for the definitions of the relative principal curvatures, written ${ }_{r} k_{1}, \ldots,{ }_{r} k_{n-1}$, and those of the relative principal radii of curvature written, ${ }_{r} r_{1}, \ldots,{ }_{r} r_{n-1}$, of the pair $(B, K)$. Actually Leichtweiss works with a gauge body of class $C_{+}^{3}$, but all the results we need holed in the $C_{+}^{2}$ case (see Chakerian [5, p.32]). We will denote by ${ }_{r} s_{j}$ the $j$ th normalised elementary symmetric function of the relative principal radii of curvature.

A point of bd $K$ is a relative umbilic point if all its relative principal curvatures are equal.

Definition 8.1. Suppose that $B$ is a centrally symmetric convex body in $\mathbb{R}^{n}$ with center at the origin.

a) A convex body $K$ has constant relative width if the ratio $\frac{\lambda_{1}(K \mid E)}{\lambda_{1}(B \mid E)}$ is constant for any line $E$ of $G(1, n)$.

b) A convex body $K$ has constant relative $j$-brightness if the ratio $\frac{\lambda_{j}(K \mid E)}{\lambda_{j}(B E)}$ is constant for any $j$-dimensional plane $E$ of $G(j, n)$.

c) The relative $k$-girth of $K \in \mathcal{K}^{n}$ in the direction $u \in \mathbb{S}^{n-1}$ is equal $([5, \S 3])$ to the mixed volume $n V(K, \ldots, K, B, \ldots, B,[u])$ where $K$ appears $k$ times, $B(n-k-1)$ times and where $[u]$ is a line segment of unit length parallel to $u$.

d) A convex body $K \in \mathcal{K}^{n}$ has constant relative $k$-girth if the ratio of the relative $k$-girth of $K \in \mathcal{K}^{n}$ in the direction $u$ to that of $B$ in the same direction is constant for all $u \in \mathbb{S}^{n-1}$.

Theorem 8.2. Let $B \in \mathcal{K}^{n}$ be a gauge body of class $C_{+}^{2}$ and let $K \in \mathcal{K}^{n}$ be a convex body of class $C_{+}^{2}$.

a) ([5, Theorem 5]) If $K$ has constant relative width equal to $b$, the relative principal radii of curvature of $K$ (with the usual convention $\left.{ }_{r} r_{1}^{\uparrow}(u) \leq{ }_{r} r_{2}^{\uparrow}(u) \leq \cdots \leq{ }_{r} r_{n-1}^{\uparrow}(u)\right)$ satisfy

$$
{ }_{r} r_{i}^{\uparrow}(u)+{ }_{r} r_{n-i}^{\uparrow}(-u)=b, \text { for all } u \in \mathbb{S}^{n-1} \text { and } 1 \leq i \leq n-1 .
$$

b) If $K$ has constant relative $j$-girth, then

$$
{ }_{r} s_{j}(u)+{ }_{r} s_{j}(-u) \quad i s \text { constant for all } u \in \mathbb{S}^{n-1} .
$$


Part b) of the theorem is a consequence of the corollary of Theorem 2 of [5] and of Theorem 4 of [5].

Let $K_{\ell}$ be convex bodies in $\mathbb{R}^{n}$ for $\ell=1, \ldots, n$ and let $1 \leq j \leq i \leq n$ be integers. If $K_{1}=K_{3}=\cdots=K_{j+1}$ and if $K_{2}=K_{j+2}=\cdots=K_{i}$, we will denote by $V\left(K_{1}, i, K_{2}, i-j, \mathcal{A}\right)$ the mixed volume where $K_{1}$ appears $i$ times, $K_{2}$ appears $i-j$ times and where $\mathcal{A}=\left\{K_{i+1}, \ldots, K_{n}\right\}$. Now we will prove a result similar to Theorem $B$ for relative differential geometry:

Theorem 8.3. Let $B \in \mathcal{K}^{n}$ be a centrally symmetric gauge body of class $C_{+}^{2}$, let $K \in \mathcal{K}^{n}$ be a convex body of class $C_{+}^{2}$ having two relative umbilic points belonging to two parallel support hyperplanes, and let $1 \leq i<n-1$ be an integer. If $K$ has both constant relative $i$ - and $(n-1)$-girth, then $K$ is a relative ball, i.e., homothetic to the gauge body $B$.

Proof. Let $V_{i}(K, B,[u])$ be $V(K, i, B, n-1-i,[u])$. If $K$ has constant $i$-girth, then there exists a constant $c_{i}$ such that for all $u \in \mathbb{S}^{n-1}$,

$$
V_{i}(K, B,[u])=c_{i} V_{0}(K, B,[u])=V_{0}\left(K, c_{i}^{\frac{1}{n-1}} B,[u]\right) .
$$

If we choose $\left(2 c_{i}\right)^{\frac{1}{n-1}} B$ as gauge body, we have ${ }_{r} s_{i}(u)+{ }_{r} s_{i}(-u)=1$ by the corollary of Theorem 2 of [5]. Consequently if the gauge body is $B$,

$$
{ }_{r} s_{i}(u)+{ }_{r} s_{i}(-u)=2 c_{i}, \quad \text { for all } u \in \mathbb{S}^{n-1} .
$$

We choose $u_{0} \in \mathbb{S}^{n-1}$ such that $\nu^{-1}\left(u_{0}\right)$ and $\nu^{-1}\left(-u_{0}\right)$ are relative umbilic points. We will denote their relative radii of curvature by $x$ and $y$. Suppose that $K$ has constant $i$ - and $j$-girth. The equalities (16) and (17) are true for $i$ and $j$. Thus, if $i<j$, we have by Jensen's inequality:

$$
\begin{aligned}
\left(\frac{V_{i}(K, B,[u])}{V_{0}(K, B,[u])}\right)^{\frac{1}{i}} & =\left(\frac{1}{2}\left({ }_{r} s_{i}\left(u_{0}\right)+{ }_{r} s_{i}\left(-u_{0}\right)\right)\right)^{\frac{1}{i}} \\
& =\left(\frac{1}{2}\left(x^{i}+y^{i}\right)\right)^{\frac{1}{i}} \\
& \leq\left(\frac{1}{2}\left(x^{j}+y^{j}\right)\right)^{\frac{1}{j}} \\
& =\left(\frac{1}{2}\left(r s_{j}\left(u_{0}\right)+{ }_{r} s_{j}\left(-u_{0}\right)\right)\right)^{\frac{1}{j}} \\
& =\left(\frac{V_{j}(K, B,[u])}{V_{0}(K, B,[u])}\right)^{\frac{1}{j}} .
\end{aligned}
$$


On the other hand, the Aleksandrov-Fenchel inequality ([10, (B.16)]) implies

$$
V_{i}(K, B,[u])^{n-1} \geq V_{n-1}(K, B,[u])^{i} V_{0}(K, B,[u])^{n-1-i} .
$$

Consequently $V_{i}(K, B,[u])^{\frac{1}{i}} \geq V_{n-1}(K, B,[u])^{\frac{1}{n-1}} V_{0}(K, B,[u])^{\left(\frac{1}{i}-\frac{1}{n-1}\right)}$, i.e.,

$$
\left(\frac{V_{i}(K, B,[u])}{V_{0}(K, B,[u])}\right)^{\frac{1}{i}} \geq\left(\frac{V_{n-1}(K, B,[u])}{V_{0}(K, B,[u])}\right)^{\frac{1}{n-1}} .
$$

Inequality (18) is, by hypothesis, satisfied for $j=n-1$. It implies that inequality (20), and therefore inequality (19), are in fact equalities.

We know that

$$
V_{i}(K, B,[u])=n^{-1} V\left(\left(K \mid u^{\perp}\right), i,\left(B \mid u^{\perp}\right), n-1-i\right)
$$

by $([5, \mathrm{p} .28])$, that

$$
V_{0}(K, B,[u])=n^{-1} V\left(B \mid u^{\perp}, n-1\right)=n^{-1} \lambda_{n-1}\left(B \mid u^{\perp}\right)
$$

and that

$$
V_{n-1}(K, B,[u])=n^{-1} V\left(K \mid u^{\perp}, n-1\right)=n^{-1} \lambda_{n-1}\left(K \mid u^{\perp}\right) .
$$

Therefore the inequality

$$
\begin{aligned}
V\left(\left(K \mid u^{\perp}\right), i,\left(B \mid u^{\perp}\right), n-i-1\right)^{n-1} \\
\quad \geq V\left(\left(K \mid u^{\perp}\right), n-1\right)^{i} V\left(\left(B \mid u^{\perp}\right), n-1\right)^{n-1-i}
\end{aligned}
$$

is in fact an equality. This implies that, for all $u \in \mathbb{S}^{n-1}, K \mid u^{\perp}$ and $B \mid u^{\perp}$ are homothetic by the observations on the cases of equality of (B.16) on page 372 of [10]. Consequently $K$ and $B$ are homothetic by Theorem 3.1.3 of [10]. q.e.d.

Theorem 8.4. Let $\left(X,\|\|_{B}\right)$ be a Minkowski space whose gauge body $B$ is of class $C_{+}^{2}$. Any convex body $K \subset X$ of class $C_{+}^{2}$, which has constant Minkowski width and constant Minkowski brightness is homothetic to the gauge body $B$.

Proof. We proceed as for Theorem 7.1 but using relative principal radii of curvature. We conclude using Formula (4.4) of [5] or Theorem 6.2 of $[15]$. q.e.d. 
Theorem 8.5. A convex body $K$ of class $C_{+}^{2}$ which has constant relative width and constant relative $k$-brightness for a given $k>1$, is a ball.

Proof. We proceed as for Theorem 7.2 and we conclude using Theorem 3.1.3 of [10]. q.e.d.

Let $K, L \in \mathcal{K}^{n}$ be of class $C_{+}^{2}$, and let $J:$ bd $K \rightarrow$ bd $L$ be the canonical map sending the point $x \in \mathrm{bd} K$ to the point $J(x) \in \mathrm{bd} L$ with the same normal as $x$. This is Petersen's map (see [15, p. 1075]; $[5$, p. 31]).

Theorem 8.6. Suppose that $K, L$, are convex bodies in $\mathbb{R}^{n}$ of class $C_{+}^{2}$. If the gauge body $L$ is centrally symmetric and if $K$ has constant relative $k$-brightness, we have for all $x \in \mathrm{bd} L$, that $\wedge^{k} J^{-1}(x)+\wedge^{k} J^{-1}(-x)$ is a scalar multiple of the identity of $\wedge^{k} T_{x} \mathrm{bd} L$, where $J$ denotes Petersen's map, and $-x$ the unique point of $\mathrm{bd} L$ whose tangent hyperplane is parallel to that at $x$.

Proof. We proceed as for Theorem 4.1. q.e.d.

Identical reasoning to that of Theorem E leads to

Theorem 8.7. Suppose there are two convex bodies $K, L \in \mathcal{K}^{n}$ $(n \geq 4)$ of class $C_{+}^{2}$, where the gauge body $L$ is centrally symmetric. If $K$ has constant relative $k$-brightness for a given integer $k$ where $1<k<n-2$ or $K$ has both constant relative 1 - and $(n-2)$-brightness, then the hypersurface $\mathrm{bd} K$ has at least one pair of relative umbilic points belonging to two parallel support hyperplanes.

Theorem B. Let two convex bodies $K, L$ in $\mathbb{R}^{n}$ be of class $C_{+}^{2}$, where $L$ is centrally symmetric. If, for two distinct values of $i(1 \leq i<n)$, the ratio of the volumes of their projections on any $i$-dimensional subspace is a constant (depending on $i$ ), then $K$ and $L$ are homothetic.

Proof. If $(i, j)=(1, n-1)$, we know that the hypersurface bd $K$ is a relative ball by Theorem 8.4 .

If $(i, j) \neq(1, n-1)$, the hypersurface bd $K$ has a pair of relative umbilic points belonging to two parallel support hyperplanes by Theorem 8.7. If $i<j$, the convex bodies $K \mid E$ and $L \mid E$ are of class $C_{+}^{2}$ for all $E \in G(n, j+1)$. The convex body $K \mid E$ has a pair of umbilic points (relative to the gauge body $L \mid E$ ). Theorem 8.3 enables us to deduce that $K \mid E$ and $L \mid E$ are homothetic for all $E \in G(n, j+1)$. Consequently $K$ and $L$ are homothetic by Theorem 3.1 .3 of [10]. q.e.d. 


\section{Linear relations between $k$-girths in direction $u$ for a body of constant width}

We recall some combinatorial results.

The binomial coefficients $\left(\begin{array}{c}-n \\ p\end{array}\right)$, where $n \in \mathbb{N}$ and $p \in \mathbb{N}$, are defined $([19, \S 156(1)])$ by

$$
\left(\begin{array}{c}
-n \\
p
\end{array}\right)=(-1)^{p}\left(\begin{array}{c}
n+p-1 \\
p
\end{array}\right)
$$

Up to sign, this is equal to the number of way of choosing $p$ elements among $n$ with repetitions allowed. We know that, for all $p \in \mathbb{N}$,

$$
\left(\begin{array}{l}
n \\
p
\end{array}\right)=0 \quad \text { if } 0<n<p, \quad\left(\begin{array}{c}
n \\
m
\end{array}\right)=\left(\begin{array}{c}
n \\
n-m
\end{array}\right),
$$

and

$$
\left(\begin{array}{l}
n \\
m
\end{array}\right)\left(\begin{array}{c}
m \\
p
\end{array}\right)=\left(\begin{array}{l}
n \\
p
\end{array}\right)\left(\begin{array}{c}
n-p \\
m-p
\end{array}\right)=\left(\begin{array}{c}
n \\
m-p
\end{array}\right)\left(\begin{array}{c}
n-m+p \\
p
\end{array}\right)
$$

and we recall the combinatorial identity $([19, \S 164(42)$ and $§ 158(25)])$

$$
\sum_{s=0}^{q}(-1)^{s}\left(\begin{array}{c}
m \\
q-s
\end{array}\right)\left(\begin{array}{c}
n+s \\
n
\end{array}\right)=\left(\begin{array}{c}
m-n-1 \\
q
\end{array}\right) .
$$

Let $a=\left(a_{1}, \ldots, a_{n}\right)$ and $b=\left(b_{1}, \ldots, b_{n}\right)$ be $n$-tuples of real numbers, and let $a-b$ be the $n$-tuple $\left(a_{1}-b_{1}, \ldots, a_{n}-b_{n}\right)$. For any integer $j$ $(1 \leq j \leq n)$, we will denote by $S_{j}(a)$ or $S_{j}^{(n)}(a)$ the $j$ th elementary symmetric function in $a$ and by $s_{j}(a)$ or $s_{j}^{(n)}(a)$ the $j$ th normalised elementary symmetric function in $a$. Let $s_{0}(a)=S_{0}(a)=1$.

In this section we may suppose without loss of generality, by applying a homothety, if necessary, that the width of the convex body $K \in$ $\mathcal{W}^{n}$ is equal to one. We recall that $S_{j}^{(n-1)}(u)=\left(\begin{array}{c}n-1 \\ j\end{array}\right) s_{j}(u)$ is the $j$ th elementary symmetric function $S_{j}^{(n-1)}\left(r^{\uparrow}(u)\right)$ of the principal radii of curvature of $K$ at $u$. We have $r^{\uparrow}(u)+r^{\downarrow}(-u)=(1, \ldots, 1)$ by Theorem 2.2. Consequently

$$
\begin{aligned}
\left(\begin{array}{c}
n-1 \\
j
\end{array}\right) s_{j}(-u) & =S_{j}^{(n-1)}\left(r^{\downarrow}(-u)\right)=\sum_{i=0}^{j}(-1)^{i}\left(\begin{array}{c}
n-1-i \\
n-1-j
\end{array}\right) S_{i}^{(n-1)}\left(r^{\uparrow}(u)\right) \\
& =\sum_{i=0}^{j}(-1)^{i}\left(\begin{array}{c}
n-1-i \\
n-1-j
\end{array}\right)\left(\begin{array}{c}
n-1 \\
i
\end{array}\right) s_{i}(u) .
\end{aligned}
$$


Therefore, as $\left(\begin{array}{c}n-1-i \\ n-1-j\end{array}\right)=0$ for $0 \leq j<i \leq n-1$, we have

$$
S_{j}^{(n-1)}(-u)=\sum_{i=0}^{n-1}(-1)^{i}\left(\begin{array}{c}
n-1-i \\
n-1-j
\end{array}\right) S_{i}^{(n-1)}(u) .
$$

For any convex body $K \in \mathcal{K}^{n}$ of class $C_{+}^{2}$, the functions

$$
P_{j}^{K}: \mathbb{S}^{n-1} \rightarrow \mathbb{R} \quad(0 \leq j \leq n-1)
$$

defined by $P_{j}^{K}(u)=S_{j}^{(n-1)}(u)+S_{j}^{(n-1)}(-u)$ represent, up to a constant multiplicative factor, the $k$-girth of $K \in \mathcal{K}^{n}$ in the direction $u \in \mathbb{S}^{n-1}$. Let $P_{j}^{K} \equiv 0$ if $j<0$ or $j>n-1$.

Lemma 9.1. Let $n$ and $a$ be integers with $n-1 \geq 2 a \geq 2$. If $K \in \mathcal{W}^{n+1}$ is of class $C_{+}^{2}$, the $(a+2)$ continuous functions

$$
P_{0}^{K}, P_{a+1}^{K}, P_{a+2}^{K}, \ldots, P_{2 a+1}^{K}
$$

or

$$
P_{0}^{K}, P_{2}^{K}, P_{a+2}^{K}, P_{a+3}^{K}, \ldots, P_{2 a+1}^{K}
$$

are linearly dependent.

Proof. For any integer $b \leq a$, let

$$
A_{j, 2 a+1-j}(b)=(-1)^{j}\left(\begin{array}{c}
2 b-j \\
b
\end{array}\right)\left(\begin{array}{c}
n-2 a-1+j \\
n-2 a-1
\end{array}\right) .
$$

The linear combination $\sum_{j=0}^{b} A_{j, 2 a+1-j}(b) S_{2 a+1-j}^{(n)}(-u)$ is equal to

$$
\begin{aligned}
\sum_{j=0}^{b} \sum_{i=0}^{2 a+1}(-1)^{i+j}\left(\begin{array}{c}
2 b-j \\
b
\end{array}\right)\left(\begin{array}{c}
n-2 a-1+j \\
n-2 a-1
\end{array}\right)\left(\begin{array}{c}
n-i \\
n-2 a-1+j
\end{array}\right) S_{i}^{(n)}(u) \\
\quad=\sum_{j=0}^{b} \sum_{i=0}^{2 a+1}(-1)^{i+j}\left(\begin{array}{c}
2 b-j \\
b
\end{array}\right)\left(\begin{array}{c}
n-i \\
n-2 a-1
\end{array}\right)\left(\begin{array}{c}
2 a+1-i \\
j
\end{array}\right) S_{i}^{(n)}(u) \\
\quad=\sum_{i=0}^{2 a+1}(-1)^{i}\left(\begin{array}{c}
n-i \\
n-2 a-1
\end{array}\right)\left(\begin{array}{c}
2(b-a)+i-1 \\
b
\end{array}\right) S_{i}^{(n)}(u) \\
=\left[\sum_{i=0}^{2(a-b)}+\sum_{i=a+1}^{2 a+1}\right](-1)^{i}\left(\begin{array}{c}
n-i \\
n-2 a-1
\end{array}\right)\left(\begin{array}{c}
2(b-a)+i-1 \\
b
\end{array}\right) S_{i}^{(n)}(u) \\
\quad=\left[-\sum_{j=2 b+1}^{2 a+1}-\sum_{j=0}^{a}\right] A_{j, 2 a+1-j}(b) S_{2 a+1-j}^{(n)}(u),
\end{aligned}
$$


by using successively (25), (23), (24) and (22). Consequently

$$
\sum_{j=0}^{b} A_{j, 2 a+1-j}(b) P_{2 a+1-j}^{K}(u)=-\sum_{j=2 b+1}^{2 a+1} A_{j, 2 a+1-j}(b) S_{2 a+1-j}^{(n)}(u)
$$

If $b=a,(26)$ shows that the functions $P_{0}^{K}, P_{a+1}^{K}, P_{a+2}^{K}, \ldots, P_{2 a+1}^{K}$ are linearly dependent.

If $b=a-1$, the right-hand side of (26) is, by (21), equal to

$$
\begin{gathered}
(-1)^{a}\left[\left(\begin{array}{c}
n-2 \\
2 a-1
\end{array}\right) S_{2}^{(n)}(u)-\left(\begin{array}{c}
a \\
a-1
\end{array}\right)\left(\begin{array}{c}
n-1 \\
2 a
\end{array}\right) S_{1}^{(n)}(u)+\left(\begin{array}{c}
a+1 \\
a-1
\end{array}\right)\left(\begin{array}{c}
n \\
2 a+1
\end{array}\right) S_{0}^{(n)}(u)\right] \\
=(-1)^{a}\left[\frac{1}{2}\left(\begin{array}{c}
n-2 \\
2 a-1
\end{array}\right) P_{2}^{K}(u)-\frac{a^{2}}{2}\left(\begin{array}{c}
n \\
2 a+1
\end{array}\right) P_{0}^{K}(u)\right],
\end{gathered}
$$

because as $\frac{1}{2}\left(\begin{array}{c}n-1 \\ n-2\end{array}\right)\left(\begin{array}{c}n-2 \\ 2 a-1\end{array}\right)=\frac{1}{2}\left(\begin{array}{c}n-1 \\ n-2 a-1\end{array}\right)\left(\begin{array}{c}2 a \\ 2 a-1\end{array}\right)=\left(\begin{array}{c}n-1 \\ 2 a\end{array}\right)\left(\begin{array}{c}a \\ a-1\end{array}\right)$ by (23), we have, by $(25)$,

$$
\begin{gathered}
\frac{1}{2}\left(\begin{array}{c}
n-2 \\
2 a-1
\end{array}\right) P_{2}^{K}(u)=\left(\begin{array}{c}
n-2 \\
2 a-1
\end{array}\right)\left[S_{2}^{(n)}(u)-\frac{1}{2}\left(\begin{array}{c}
n-1 \\
n-2
\end{array}\right) S_{1}^{(n)}(u)+\frac{1}{2}\left(\begin{array}{c}
n \\
2
\end{array}\right) S_{0}^{(n)}(u)\right] \\
=\left(\begin{array}{c}
n-2 \\
2 a-1
\end{array}\right) S_{2}^{(n)}(u)-\left(\begin{array}{c}
a \\
a-1
\end{array}\right)\left(\begin{array}{c}
n-1 \\
2 a
\end{array}\right) S_{1}^{(n)}(u)+\frac{1}{2}\left(\begin{array}{c}
n-2 \\
2 a-1
\end{array}\right)\left(\begin{array}{c}
n \\
2
\end{array}\right) S_{0}^{(n)}(u) .
\end{gathered}
$$

This proves that the functions $P_{0}^{K}, P_{2}^{K}, P_{a+2}^{K}, \ldots, P_{2 a+1}^{K}$ are linearly dependent. q.e.d.

Lemma 9.2. Let $a, b$ and $n$ be integers with $n-1 \geq 2 a \geq 2$ and $0 \leq b \leq a$. If $K \in \mathcal{W}^{n+1}$ is of class $C_{+}^{2}$, the $(a+2)$ continuous functions $P_{0}^{K}, P_{2}^{K}, \ldots, P_{2 b}^{K}, P_{a+b+1}^{K}, P_{a+b+2}^{K}, \ldots, P_{2 a+1}^{K}$ are linearly dependent.

Proof. We proceed by induction on $a$. If $a=1$ or 2 , it is a special case of Lemma 9.1. In order to establish the induction step from $a$ to $a+1$, we proceed again by induction on $b$, using Lemma 9.1 with $b=0$ or 1. q.e.d.

Corollary 9.3. Let $a$ and $n$ be integers such as $n-1 \geq 2 a \geq 2$ and let $K \in \mathcal{W}^{n+1}$ be of class $C_{+}^{2}$. For any choice of $a-1$ distinct integers $i_{j}$ where $2 \leq i_{j} \leq 2 a-1$, the $a+2$ continuous functions $P_{0}^{K}, P_{i_{1}}^{K}, \ldots, P_{i_{a-1}}^{K}$, $P_{2 a}^{K}, P_{2 a+1}^{K}$ are linearly dependent. 
After integration, these linear relations resemble those betweeen quermassintegrals $W_{i}=W_{i}(K)$ of a convex body $K \in \mathcal{W}^{n}$, quoted in [21], Section 5.3, Note 4 and in [6]. However, we have observed that they do not coincide, even if they have a common intersection. It would be interesting to analyse more closely these two sequences of relations.

Theorem 9.4. Let $K \in \mathcal{W}^{n+1}$ be of class $C_{+}^{2}$.

a) Let $a$ and $n$ be integers such that $n-1 \geq 2 a \geq 4$. If $K$ has constant $2 a$-girth and constant $i_{j}$-girth for $a-1$ distinct integers $i_{j}$ where $2 \leq i_{j} \leq 2 a-1$, then $K$ has constant $(2 a+1)$-girth.

b) If $K$ has constant 2-girth, $K$ also has constant 3 -girth.

Proof. This a direct consequence of Corollary 9.3 and Theorem 2.4 a). q.e.d.

Theorem $\mathbf{C}$. Let $K$ be a convex body in $\mathbb{R}^{2 n}$ of class $C_{+}^{2}$ and constant width.

a) If $2 n \geq 6$ and if $K$ has constant $(2 n-2)$-girth and constant $i_{j}$ girth for $n-2$ distinct integers $i_{j}$ with $2 \leq i_{j} \leq 2 n-3$, then $K$ is a ball.

b) If $n=2$ and $K$ has constant 2-girth, then $K$ is a ball.

Proof. We recall that a convex body in $\mathbb{R}^{2 n}$ of constant $(2 n-1)$ girth has constant $(2 n-1)$-brightness by Theorem $2.3 \mathrm{c}$ ). We conclude by using Theorem A. q.e.d.

\section{References}

[1] D. W. Barnes, On the existence of umbilics on compact surfaces, Arch. Math. 18 (1967) 320-324.

[2] V. G. Boltyanski, H. Martini \& P. S. Soltan, Excursions into combinatorial geometry, Springer, Berlin, 1997.

[3] T. Bonnesen \& W. Fenchel, Theory of convex bodies, BCS Associates (Moscow, U. S.), 1987 transl. by L. Boron, C. Christensen and B. Smith of Theorie der konvexen Körper, Springer, Berlin, 1934.

[4] G. E. Bredon, Topology and geometry, Graduate Texts in Math. 139, Springer, New York, 1993.

[5] G. D. Chakerian, Sets of constant relative width and constant relative brightness, Trans. Amer. Math. Soc. 129 (1967) 26-37. 
[6] G. D. Chakerian \& H. Groemer, Convex bodies of constant width, Convexity and its applications, (ed. by P. M. Gruber and J. M. Wills), Birkhäuser, Basel, 1983, $49-96$.

[7] H. T. Croft, K. J. Falconer \& R. K. Guy, Unsolved Problems in Geometry, Springer, New York, 1991.

[8] E. A. Feldman, On parabolic and umbilic points of hypersurfaces, Trans. Amer. Math. Soc. 127 (1967) 1-28.

[9] W. F. Firey, Convex bodies of constant outer p-measure, Mathematika 17 (1970) 21-27.

[10] R. J. Gardner, Geometric tomography, Cambridge University Press, Cambridge, 1995 .

[11] R. J. Gardner \& A. volčič, Tomography of convex and star bodies, Adv. Math. 108 (1994) 367-399.

[12] P. Goodey, R. schneider \& W. Weil, On the determination of convex bodies by projection functions, Bull. London Math. Soc. 29 (1997) 82-88.

[13] Projection functions of convex bodies, Bolyai Society Math. Stud. 6 (1997) 23-53.

[14] E. Heil \& H. Martini, Special convex bodies, Handbook of Convex Geometry, (ed. by P. M. Gruber and J. M. Wills), Elsevier, Amsterdam, 1993, 347-385.

[15] K. Leichtweiss, Convexity and differential geometry, Handbook of Convex Geometry, (ed. by P. M. Gruber and J. M. Wills), Elsevier, Amsterdam, 1993, $1045-1080$.

[16] M. Marcus \& H. Minc, A survey of matrix theory and matrix inequalities, Prindle, Weber \& Schmidt, Boston, 1964.

[17] A. Marshall \& I. Olkin, Inequalities: Theory of Majorization and its applications, Academic Press, New York, 1979.

[18] S. Nakajima, Eine charakteristische Eigenschaft der Kugel, Jber. Deutsche Math.Verein. 35 (1926) 298-300.

[19] E. Netto, Lehrbuch der Combinatorik, $2^{\text {nd }}$ ed., Teubner, Leipzig, 1927; $3^{d}$ ed., Chelsea, New York.

[20] A. M. Ostrowski, Sur quelques applications des fonctions convexes et concaves au sens de I. Schur, J. Math. Pures Appl. (9) 31 (1952) 253-292.

[21] R. Schneider, Convex bodies: The Brunn-Minkowski theory, Cambridge University Press, Cambridge, 1993.

[22] M. Spivak, A Comprehensive introduction to differential geometry, Vol. III, $2^{\text {nd }}$ ed., Publish or Perish, Berkeley, CA, 1979. 
[23] A. C. Thompson, Minkowski geometry, Cambridge University Press, Cambridge, 1996.

Institut de Mathématiques, BCH UNIVERSiTÉ DE LAUSANNE, SWITZERLAND 